Supporting Information for:

\title{
Determination of Crystal-Field Splitting Induced by Thermal
}

\section{Oxidation of Titanium}

Klaudia Wojtaszek ${ }^{1}$, Wojciech Błachucki ${ }^{1}$, Krzysztof Tyrała ${ }^{1}$, Michał Nowakowski ${ }^{1,2}$, Marcin Zając $^{3}$, Joanna Stępień ${ }^{4}$, Paweł Jagodziński ${ }^{5}$, Dariusz Banaśs ${ }^{5}$, Wiktoria Stańczyk ${ }^{1}$, Joanna

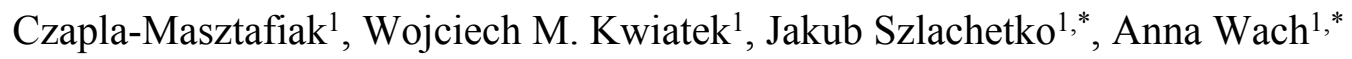

${ }^{1}$ Institute of Nuclear Physics Polish Academy of Sciences, 31-342 Krakow, Poland

${ }^{2}$ Universität Paderborn, Department Chemie, Warburger Str. 100, Paderborn 33098, Germany

${ }^{3}$ National Synchrotron Radiation Centre Solaris, Jagiellonian University, Krakow, Poland

${ }^{4}$ Academic Centre for Materials and Nanotechnology AGH, Krakow, Poland

${ }^{5}$ Institute of Physics, Jan Kochanowski University, 25-406 Kielce, Poland

*Corresponding authors: anna.wach@ifj.edu.pl; jakub.szlachetko@ifj.edu.pl

\section{Table of Contents}

Section S1 - The O K-edge XAS spectra collected for all studied samples: $\quad$ S2

$\begin{array}{ll}\text { Section S2 - The XAS fitting procedure and error analysis details: } & \text { S3 }\end{array}$

$\begin{array}{ll}\text { Section S3 - Crystal structure determination by XRD } & \text { S4 }\end{array}$

$\begin{array}{ll}\text { Section } \mathbf{S 4} \text { - Surface chemical state analysis by XPS: } & \text { S6 }\end{array}$ 
Section S1 - The O K-edge XAS spectra collected for all studied samples:

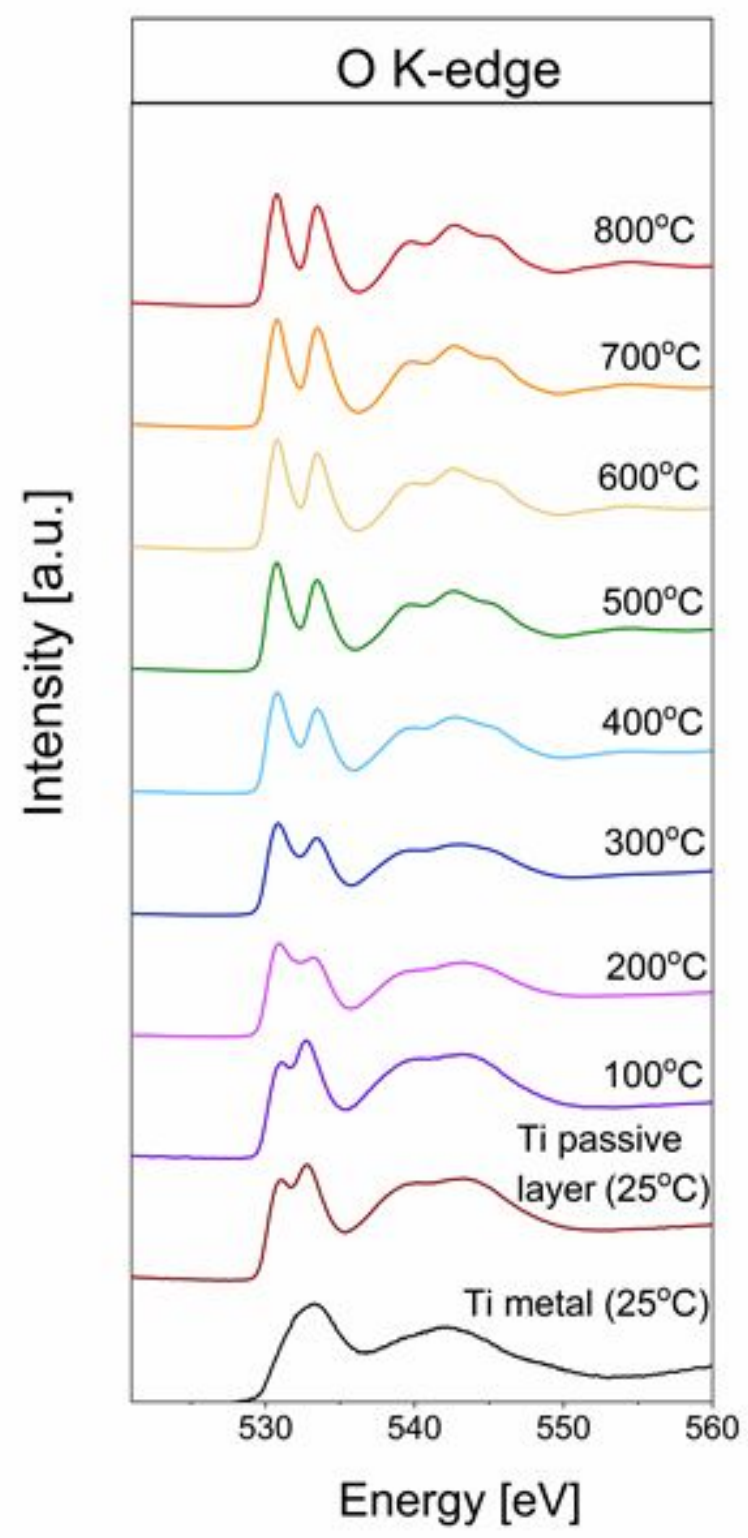

Fig. S1. The O K-edge XAS spectra collected for metallic Ti disc, Ti disc with passive oxide layer $\left(25^{\circ} \mathrm{C}\right)$ and Ti discs thermally oxidized in ambient atmosphere at different temperatures. 
Section S2 - The XAS fitting procedure and error analysis details:

\section{Determination of $\Delta$ oct and $t_{2 g} / e_{g}$ from the Ti $L_{2}$-edge XAS spectra}

The spectra were fitted with Gaussian peaks using the least squares method.

$\Delta$ oct was obtained through subtraction of the relevant peaks' (structures $\mathrm{d}$ and $\mathrm{f}$ in the text, respectively) positions, $p_{1}$ and $p_{2}$ :

$$
\Delta \text { oct }=p_{2}-p_{1}
$$

The $\mathrm{t}_{2 \mathrm{~g}} / \mathrm{e}_{\mathrm{g}}$ was obtained through division of the relevant peaks' (structures $\mathrm{d}$ and $\mathrm{f}$ in the text, respectively) amplitudes $a m p_{1}$ and $a m p_{2}$ :

$$
\mathrm{t}_{2 \mathrm{~g}} / \mathrm{e}_{\mathrm{g}}=\operatorname{amp}_{1} / \mathrm{amp}_{2} \text { : }
$$

The error of $\Delta$ oct was calculated in accordance with the propagation of uncertainty:

$$
\operatorname{error}(\Delta \mathrm{oct})=\sqrt{\operatorname{error}\left(p_{1}\right)^{2}+\operatorname{error}\left(p_{2}\right)^{2}}
$$

with $\operatorname{error}\left(p_{1}\right)$ and $\operatorname{error}\left(p_{2}\right)$ being the standard errors of the peaks' positions' estimates obtained in the fitting procedure.

The error of $\mathrm{t}_{2 \mathrm{~g}} / \mathrm{e}_{\mathrm{g}}$ was calculated with the formula

$$
\operatorname{error}\left(t_{2 g} / e_{g}\right)=\frac{a m p_{1}}{a m p_{2}} \sqrt{\left(\frac{\operatorname{error}\left(a m p_{1}\right)}{a m p_{1}}\right)^{2}+\left(\frac{\operatorname{error}\left(a m p_{2}\right)}{a m p_{2}}\right)^{2}}
$$

with $a m p_{1}$ and $a m p_{2}$ being the peaks' amplitude's estimates and their standard errors: $\operatorname{error}\left(a m p_{1}\right)$ and $\operatorname{error}\left(a m p_{2}\right)$.

\section{Determination of $\Delta$ oct and $t_{2 g} / e_{g}$ from the $T i L_{3}$-edge XAS spectra}

$\Delta$ oct and the $\mathrm{t}_{2 \mathrm{~g}} / \mathrm{e}_{\mathrm{g}}$ ratio were extracted from the $\mathrm{Ti}_{\mathrm{L}}$-edge XAS spectra from the structures $\mathrm{b}$ and $\mathrm{c}_{1,2}$ as described above with one difference: while in the Ti $\mathrm{L}_{2}$-edge XAS spectra the electronic transition $2 \mathrm{p}_{1 / 2} \rightarrow \mathrm{e}_{\mathrm{g}}$ is represented by a single peak, denoted $\mathrm{f}$ in the text, in the $\mathrm{Ti}$ $\mathrm{L}_{3}$-edge XAS spectra the electronic transition $2 \mathrm{p}_{3 / 2} \rightarrow \mathrm{e}_{\mathrm{g}}$ is split into two peaks, namely $\mathrm{c}_{1}$ and $\mathrm{c}_{2}$. These two peaks were fitted separately, yielding their positions $p_{c_{1}}, p_{c_{2}}$ and amplitudes $a m p_{c_{1}}, a m p_{c_{2}}$. Therefore, in the $\Delta$ oct and $\mathrm{t}_{2 \mathrm{~g}} / \mathrm{e}_{\mathrm{g}}$ calculations, averages of the two peaks' positions and amplitudes were used for $p_{2}$ and $a m p_{2}$ values, respectively.

$$
p_{2}=\frac{p_{c_{1}}+p_{c_{2}}}{2}, a m p_{2}=\frac{a m p_{c_{1}}+a m p_{c_{2}}}{2}
$$

hence

$$
\begin{gathered}
\operatorname{error}\left(p_{2}\right)=\frac{1}{2} \sqrt{\operatorname{error}\left(p_{c_{1}}\right)^{2}+\operatorname{error}\left(p_{c_{2}}\right)^{2}}, \\
\operatorname{error}\left(a m p_{2}\right)=\frac{1}{2} \sqrt{\operatorname{error}\left(a m p_{c_{1}}\right)^{2}+\operatorname{error}\left(a m p_{c_{2}}\right)^{2}}
\end{gathered}
$$

In turn, the values $p_{1}$ and $a m p_{1}$ were extracted from the structure $\mathrm{b}$ interpreted as $2 \mathrm{p}_{3 / 2} \rightarrow \mathrm{t}_{2 \mathrm{~g}}$ transition, which was fitted with a single peak. 
Section S3 - Crystal structure determination by XRD:

X-ray diffraction method was used to determine the crystal structure of phases formed on the surface of the titanium discs before and after thermal oxidation. XRD measurements were performed in Bragg-Brentano geometry using X'Pert Pro MPD diffractometer (PANalytical) equipped with $\mathrm{Cu}$ anode $1.8 \mathrm{~kW}$ X-ray tube. The X-rays diffracted on the analyzed sample were measured with the position-sensitive silicon strip detector (X'Celerator). Measurements were taken in the $2 \theta$ range of $20^{\circ}-70^{\circ}$, with the step size $0.0083^{\circ}$ and time per step about $45 \mathrm{~s}$. Collected XRD patterns were analyzed qualitatively with Highscore 3.0e software using PDF-2 Release 2020 database of International Centre for Diffraction Data (ICDD). The reference patterns used for the diffraction pattern interpretation were as follows: hexagonal $\alpha$-Ti (PDF 00-044-1294) and rutile $\mathrm{TiO}_{2}$ (PDF 01-072-4821). The XRD patterns collected for four samples, namely Ti disc with passive oxide layer (without any treatment) and $\mathrm{Ti}$ disc oxidized at 300,500 and $800^{\circ} \mathrm{C}$ are presented in Figure S2.

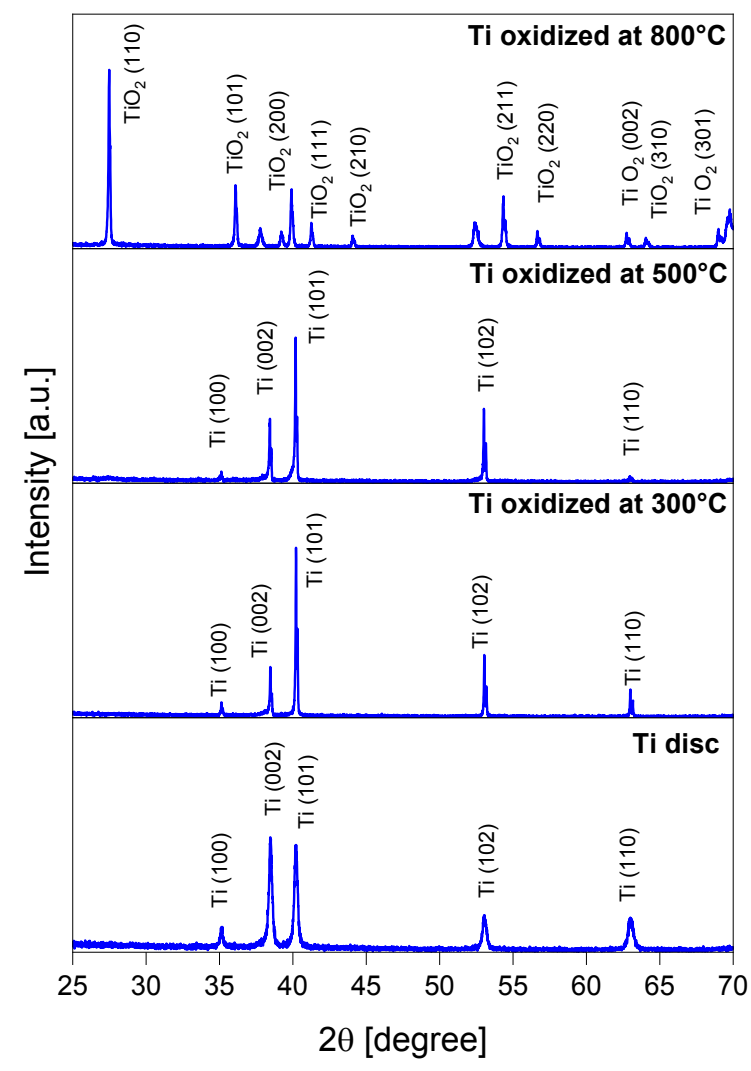

Fig. S2. X-ray diffraction patterns collected for Ti disc with passive oxide layer (without any treatment) and Ti disc oxidized at 300,500 and $800^{\circ} \mathrm{C}$. 
The XRD pattern collected for Ti disc with passive oxide layer shows sharp peaks, which can be ascribed to the metallic titanium. As shown in Fig. S2, the diffraction peaks at $2 \theta=35.1^{\circ}, 38.5^{\circ}$, $40.2^{\circ}, 53.1^{\circ}$ and $63.0^{\circ}$ are assigned to (100), (002), (101), (102) and (110) crystal planes of hexagonal $\alpha$-Ti (PDF 00-044-1294). After thermal oxidation of $\mathrm{Ti}$ discs at intermediate temperatures $\left(300\right.$ and $\left.500^{\circ} \mathrm{C}\right)$, the diffraction peaks can be entirely indexed to the metallic phase. The visible difference in the relative peak intensities may be related to the increased crystallinity of the samples after heat treatment. It should be noted that no titanium dioxide or the substoichiometric titanium oxides peaks were observed in these two samples. This is mainly due to the sampling depth of X-ray in XRD measurements, which is estimated to be 4-17 $\mu \mathrm{m}$ for diffraction angles from 20 to $70^{\circ}$. We should stress here, that according to the literature [1], the thermal oxidation of pure titanium at intermediate temperatures in an ambient atmosphere for 2 and 4 hours, results in the formation of an oxide layer with a thickness in the range of 30-50 nm. Nevertheless, the obtained XRD pattern confirmed the formation of the rutile $\mathrm{TiO}_{2}$ in the sample oxidized at $800^{\circ} \mathrm{C}$. As shown in Fig. S2, the diffraction peaks located at $2 \theta=27.5^{\circ}, 36.2^{\circ}, 39.3^{\circ}$, $41.3^{\circ}, 44.1^{\circ}, 54.4^{\circ}, 56.7^{\circ}, 62,8^{\circ}, 64.1^{\circ}$ and $69.1^{\circ}$ are indexed to (110), (101), (200), (111), (210), (211), (220), (002), (310) and (301) diffraction peaks of rutile phase (PDF. 01-072-4821, tetragonal, $\left.\mathrm{P} 4_{2} / \mathrm{mnm}\right)$. This is in agreement with literature data [2] showing that in the temperatures exceeding $600^{\circ} \mathrm{C}$, the irreversible transformation into the rutile phase is observed. 
Section S4 - Surface chemical state analysis by XPS:

To determine the composition and identify the chemical states of the titanium samples, XPS analysis was carried out. XPS spectra were recorded on SPECS monoXPS system. The system is equipped with XR50M high intensity twin anode (Al/Ag) X-ray source, FOCUS 500 quartz single crystal monochromator and PHOIBOS 100 electron analyzer with 1D delay-line detector systems (1D-DLD). The base pressure in the analysis chamber during the measurements was in the range of 6-8 $\cdot 10^{-9}$ mbar. XPS measurements were taken with a monochromatized aluminium source AlK $\alpha$ $(\mathrm{E}=1486.6 \mathrm{eV})$ and the spectra were recorded with the electron analyzer pass energy equal to $30 \mathrm{eV}$. The measured spectra were calibrated using the adventitious carbon photoelectron peak (C 1s), which energy was set to the binding energy of $284.8 \mathrm{eV}$. The composition and chemical surrounding of the sample surface were investigated on the basis of the areas and binding energies of $\mathrm{Ti} 2 \mathrm{p}, \mathrm{O} 1 \mathrm{~s}$ and $\mathrm{C} 1 \mathrm{~s}$ photoelectron peaks. The fitting of high-resolution spectra was accomplished by the CasaXPS software. The Ti 2 p core-level spectra measured by XPS for two samples: Ti disc with passive oxide layer and Ti disc oxidized at $800^{\circ} \mathrm{C}$ are shown in Figure $\mathrm{S} 3$.


Fig. S3. Ti 2p XPS spectra recorded for (A) Ti disc with passive oxide layer and (B) Ti disc oxidized at $800^{\circ} \mathrm{C}$.

It should be noted that XPS is a surface-sensitive technique, capable of providing quantitative chemical-state information from the uppermost $10 \mathrm{~nm}$. In the present study, the analysis focused on high-resolution spectra in the vicinity of the Ti $2 p$ peak. The Ti $2 p$ core-hole spectra collected 
for Ti disc with passive oxide layer, can be fitted with two doublet peaks, representing the Ti $2 p_{3 / 2}$ and Ti $2 \mathrm{p}_{1 / 2}$ states. The two peaks at $454.3 \mathrm{eV}$ and $460.3 \mathrm{eV}$ correspond to the metallic titanium. The main and dominating doublet peaks with binding energy at $457.7 \mathrm{eV}$ and $463.4 \mathrm{eV}$, are attributed to the amorphous titanium dioxide. It should be mentioned that sub-stoichiometric $\mathrm{TiO}_{\mathrm{x}}$ may be present in the sample in such small amounts that the appropriate signal can't be recognized (masked by a huge signal from $\mathrm{TiO}_{2}$ ). Quantification of the deconvoluted spectrum for the passive oxide layer on the titanium surface shows that the majority (92.27\%) of the titanium is in the form of amorphous titanium dioxide and the rest $(7.73 \%)$ is in the metallic state. The Ti $2 p$ spectra collected for Ti disc oxidized at $800^{\circ} \mathrm{C}$ can be deconvoluted with only one doublet peaks at $458.3 \mathrm{eV}$ and $464.40 \mathrm{eV}$. The peak positions and $5.7 \mathrm{eV}$ peak separation of the Ti $2 \mathrm{p}$ doublet agree well with the energy reported for $\mathrm{TiO}_{2}$ rutile phase $[3,4]$. It should be noted that no additional peak can be observed in the Ti $2 p$ spectra, which confirms the formation of crystalline form of $\mathrm{TiO}_{2}$, i.e rutile phase after thermal oxidation at $800^{\circ} \mathrm{C}$. The obtained results are in good agreement with the presented XAS data as well as the results reported in the literature. 


\section{References:}

[1] Wang, G.; Li, J.; Lv, K.; Zhang, W.; Ding, X.; Yang, G.; Liu, X.; Jiang, X. Surface thermal oxidation on titanium implants to enhance osteogenic activity and in vivo osseointegration. Sci. Rep. 2016, 6, 31769.

[2] Mehranpour, H.; Askari, M.; Sasani Ghamsari, M.; Farzalibeik, H. Study on the phase transformation kinetics of sol-gel derived TiO2 nanoparticles. J. Nanomater. 2010, 2010, 626978. [3] Pouilleau, J.; Devilliers, D.; Garrido, F.; Durand-Vidal, S.; Mah, E. Structure and composition of passive titanium oxide films. Materials Science and Engineering 1997, B47, 235-243.

[4] Oswald, S.; Gostin, P.-F.; Helth, A; Abdi, S.; Giebeler, L.; Wendrock, H.; Calin, M.; Eckerta, J.; Gebert, A. XPS and AES sputter-depth profiling at surfaces of biocompatible passivated Tibased alloys: concentration quantification considering chemical effects. Surf. Interface Anal. 2014, $46,683-688$. 